\title{
Associations of Stay-at-Home Order and Face-Masking Recommendation with Trends in Daily New Cases and Deaths of Laboratory-Confirmed COVID-19 in the United States
}

\author{
Jie Xu${ }^{1}$, Sabiha Hussain ${ }^{2}$, Guanzhu Lu${ }^{1}$, Kai Zheng ${ }^{3}$, Shi Wei ${ }^{4}$, \\ Wei Bao ${ }^{5}$ and Lanjing Zhang ${ }^{6,7,8,9^{*}}$
}

${ }^{1}$ Department of Infectious Disease, Shanghai Ninth People's Hospital, Shanghai Jiao Tong University School of Medicine, Shanghai, China ${ }^{2}$ Department of Medicine, Rutgers Robert Wood Johnson Medical School, New Brunswick, NJ, USA; ${ }^{3}$ Department of Informatics, Donald Bren School of Information and Computer Sciences, University of California, Irvine, CA, USA; ${ }^{4}$ Department of Pathology, University of Alabama at Birmingham, Birmingham, AL, USA; ${ }^{5}$ Department of Epidemiology, College of Public Health, University of Iowa, Iowa City, IA, USA; ${ }^{6}$ Department of Biological Sciences, Rutgers University Newark, NJ, USA; ${ }^{7}$ Department of Pathology, Princeton Medical Center, Plainsboro, NJ, USA; ${ }^{8}$ Department of Chemical Biology, Rutgers Ernest Mario School of Pharmacy, Piscataway, NJ, USA;

${ }^{9}$ Rutgers Cancer Institute of New Jersey, New Brunswick, NJ, USA

\begin{abstract}
Background and objectives: Public health interventions have reduced coronavirus disease 2019 (COVID-19) transmission in several countries, but their impacts on COVID-19 epidemics in the USA are unclear. We examined associations of stay-at-home order (SAHO) and face-masking recommendation with COVID-19 epidemics in the USA.
\end{abstract}

Methods: In this quasi-experimental interrupted time-series study, we modeled temporal trends in daily new cases and deaths of laboratory-confirmed COVID-19 cases, and COVID-19 time-varying reproduction numbers in the USA between March 1 and April 20, 2020. In addition, we conducted simulation analyses.

Results: The number of residents under SAHO increased since March 19 and plateaued at 290,829,980 (88.6\% of the U.S. population) on April 7. Trends in COVID-19 time-varying reproduction numbers peaked on March 23, further reduced on April 3, and fell below/around 1.0 on April 13. Early-implementation and early-lift of SAHO would reduce and increase COVID-19 epidemics, respectively. Multivariable piecewise log-linear regression revealed the states' neighboring relationship with New York was linked to COVID-19 daily new cases and deaths. There were two turning points in daily new-case trend, being March 28 (slope-changes $=-0.09$ ) and April 3 (slope-changes $=-0.09$ ), which appeared to be associated with implementation of SAHO on March 28 (affecting $48.5 \%$ of the US population in 22 states and District of Columbia), and face-masking recommendation on April 3, respectively. There were also two turning points in daily new-death trend, being April 9 (slope-changes $=-0.06$ ) and April 19 (slope-changes $=-0.90)$.

Conclusions: We identified two turning points of COVID-19 daily new cases or deaths in the USA, which seem to be linked to implementation of SAHO and the Center for Disease Control's face-masking recommendation.

Keywords: COVID; Coronavirus disease 2019; Trend; Incidence; Policy; Facemasking.

Abbreviations: CDC, Centers for Disease Control and Prevention; CI, confidence interval; COVID-19, coronavirus disease 2019; DC, District of Columbia; Rt, timevarying reproduction number; $\mathrm{SAHO}$, stay-at-home order

Received: June 25, 2020; Revised: July 03, 2020; Accepted: July 05, 2020

${ }^{*}$ Correspondence to: Lanjing Zhang, Department of Pathology, Princeton Medical Center, 1 Plainsboro Rd., Plainsboro, NJ 08563, USA. Tel: +1-609-853-6833; Fax: +1-609-85306841; E-mail: lanjing.zhang@rutgers.edu

How to cite this article: Xu J, Hussain S, Lu G, Zheng K, Wei S, Bao W, Zhang L. Associations of Stay-at-Home Order and Face-Masking Recommendation with Trends in Daily New Cases and Deaths of Laboratory-Confirmed COVID-19 in the United States. Exploratory Research and Hypothesis in Medicine 2020;5(3):77-86. doi: 10.14218/ERHM.2020.00045.

Introduction

As of July 3, 2020, the coronavirus disease 2019 (COVID-19) has been diagnosed in more than 2.7 million people and led to 128,024 deaths in the USA. ${ }^{1-3}$ Several blind spots underlying these staggering numbers have been revealed and discussed. ${ }^{4}$ In response, 40 states and the District of Columbia (DC) implemented a stay-athome order (SAHO). ${ }^{\mathbf{5}, \mathbf{6}}$ The Centers for Disease Control and Prevention (CDC) also recommended face-masking on April 3, 2020. ${ }^{7}$ 
It had been demonstrated previously that public health interventions were associated with reduction of the systemic acute respiratory syndrome-coronavirus (SARS-CoV-2) transmission in several countries ${ }^{8-11}$ but the associations of SAHO and face-masking recommendation with COVID-19 epidemics in the USA are unclear.

Therefore, we examined these associations using observed population data and then performed simulations for outcomes under the scenarios if early-implementation and lift of SAHO occurred. Without the data on these associations, the roles of SAHO and face-masking in controlling COVID-19 pandemics will remain unclear, and the public health officials and state governments will be poorly informed about the best timing, best modalities (or their combination), and best length to implement these interventions.

\section{Methods}

Quasi-experimental interrupted time series study is the comparative analysis of outcome variable(s) in a cohort with longitudinal (time series) "panel" data sets before and after an intervention. This approach is widely used in situations when randomization is not possible or extremely difficult. ${ }^{12,13}$ Due to the technical difficulty in randomization and proper control of policy implementation for COVID-19, this study design appeared to be the best fit to address the impact of COVID-19 public health interventions. Therefore, we conducted a quasi-experimental interrupted time series study to compare the changes in COVID-19 epidemics before and after SAHO and face-masking recommendation. We extracted the national and statelevel daily new cases and deaths of COVID-19 from the COVID-19 Tracking Project, which has tracked COVID-19 data since February $28,2020 .^{2}$ Only those cases and deaths that occurred from March 1 to April 20, 2020 in the 50 states and DC were analyzed.

New cases and deaths were defined as laboratory-confirmed positive cases or deaths which were reported by a state's public health authority for better data consistency and quality. ${ }^{2}$ Each of these state authorities reported its data in different format, while most, if not all, of them followed the reporting guidelines of the CDC. It is noteworthy that on April 14, 2020, the CDC updated its definition of positive cases and included probable-positive cases. ${ }^{3}$ The impact of this change to the data released on April 20, 2020 would be minimal due to the use of confirmatory laboratory-test results and the short time-interval (many states have not adopted the criteria yet). Therefore, the case/death numbers reported here might be smaller than those reported by others after April 14 .

For quality control of the released and curated data, the COVID-19 Tracking Project employed a 4-tier score system, which included four simple components, namely, reporting positive test results reliably, reporting negatives sometimes, reporting negatives reliably and reporting all commercial tests. Based on the sum of these scores, each state corresponded to a letter grade A, B, C or D, with A for the best quality. All states scored A or B.

This study was exempt (Category 4) from an Institutional Review Board review owing to the use of data from a publicly available, de-identified database.

Several population-based factors were included in the multivariable piecewise log-linear regression analyses. Specifically, the timing of SAHO and populations of the states were obtained to calculate the number of subjects and the proportion of the USA population under SAHO on a given date. ${ }^{5}$ The state populations were extracted from the USA Census (up to July 2019). ${ }^{14}$ To adjust for confounders, the proportions of daily positive results in all daily tests, and state-level daily new cases and deaths were obtained from the COVID-19 Tracking Project. ${ }^{2}$

\section{Statistical analysis}

The time-varying reproduction number $(\mathrm{Rt})$ was defined as the mean number of secondary cases generated by a typical primary case at the time $(\mathrm{t})$ in a population, and estimated using previously-reported mean serial-intervals ${ }^{15-17}$ and the R package (version 3.6.3). ${ }^{8,18}$ The mean serial interval was defined as the average time from the illness onset to successive cases in the chains of transmission. ${ }^{15-17}$ Three-day moving averages of the Rt were reported for their better sensitivity than 5-day moving averages, due to the greater amount of data points (data not shown). We estimated the segmental coefficients using piecewise log-linear models and two presumptive turning-points, including the day as the unit for $\mathrm{x}$ axis (i.e. the per-day change in the logarithmic values of COVID-19 daily new cases or deaths). Simulation analysis was performed using the prediction function. All statistical analyses were performed using Stata (version 15) and the Joinpoint program (NCI, version 4.7.0.0) with the Poisson variance option. All $p$-values were twosided, with a cut-off of 0.05 for significance.

Considering the recently reported state heterogeneity of COVID-19 epidemics in the European Union, ${ }^{19}$ we examined the association between the states' neighboring relationship with New York (epicenter at the time) and COVID-19 epidemics (daily COVID-19 new cases or new deaths). The relationship was coded as 0 for New York, 1 for the six neighbor states (Connecticut, Massachusetts, New Jersey, Pennsylvania, Rhode Island, and Vermont), and 2 for the other states and DC. We compared these epidemics using Wilcoxon rank sum test and Student's $t$-test. We then conducted sensitivity studies using multivariable piecewise log-linear regression with the states' relationship with New York as a covariate. The COVID-19 trend lines were smoothed in the figure using a locally weighted scatter smoothing (known as LOWESS) algorithm. ${ }^{20,21}$

\section{Results}

On March 19, 2020, the state of California started a SAHO which affected 39,512,223 residents (12.0\% of the U.S. population) (Fig. $1)$. The number and proportion of residents under the SAHO subsequently implemented by other 39 states and DC continued to increase until April 7, and plateaued at 290,829,980 and 88.6\%, respectively. Approximately $85.2 \%$ of the U.S. population were under SAHO on April 3, 2020, while only 3.4\% of the U.S. population were added from April 3 to April 25. The number of the states that implemented a state-wide SAHO peaked at 41 on April 25, 2020, and some states started to lift the state-wide SAHO on April 26, 2020 (Table 1).

The log-linear models used by Joinpoint program and Stata identified similar turning-points. In the multivariable piecewise log-linear regression models (Stata models), the number of states under SAHO [coefficient $=0.03,95 \%$ confidence interval $(\mathrm{CI})$ of -0.01 to $0.07, p=0.15$ ], the population under SAHO (coefficient of $\log 10$ (population under SAHO) $=-0.13,95 \%$ CI of -0.88 to $0.63, p=0.74$; coefficient of population under $\mathrm{SAHO}=0.00,95 \%$ CI of 0.00 to $0.00, p=0.78$ ), population-proportion under SAHO (coefficient $=-0.16,95 \%$ CI of -1.37 to $1.05, p=0.79$ ) and the proportion of positive tests in the USA (coefficient $=-0.24,95 \%$ CI od -0.73 to $0.24, p=0.32$ ) were not linked to the daily new cases or deaths. The trend in COVID-19 daily cases reduced after March 23 (slope changes $=-0.18,95 \%$ CI of -0.22 to $-0.14, p$ $<0.001$ ) and further reduced on April 3 (slope change $=-0.10$, $95 \%$ CI of -0.18 to $-0.08, p<0.001$ ), which appeared associated with the implementation of SAHO by 10 states (34.7\% of the U.S. population) on March 23, and the CDC's recommendation of face- 


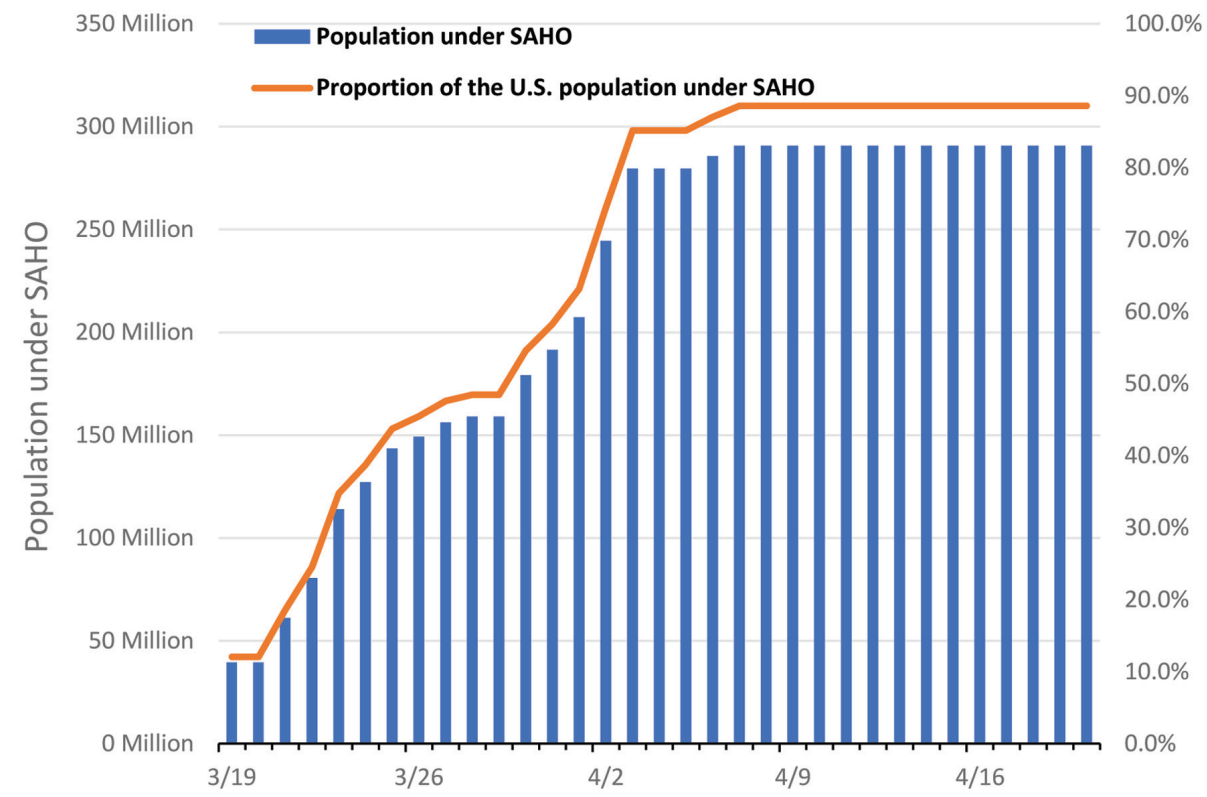

Fig. 1. The population under a stay-at-home order (SAHO) owing to the COVID-19 in the USA. Since March 19, 2020, when the state of California started a $\mathrm{SAHO}$, the number and proportion of the USA residents under SAHO increased until April 7 and plateaued afterwards.

masking on April 3, respectively (Fig. 2). Similarly, there were 2 turning-points in the trends of COVID-19 daily deaths, with a lag time of 10-12 days compared with that of COVID-19 daily new cases. The overall slope change of daily new deaths attributable to SAHO and face-masking were -0.17 (95\% CI of -0.21 to -0.14$)$ and $-0.13(95 \% \mathrm{CI}$ of -0.25 to -0.07$)$, respectively.

Our simulation analysis shows early-implementation of SAHO would be associated with a significant reduction in daily new cases and deaths, while lift of SAHO would be associated with a significant increase in daily new cases and deaths (Fig. 2). The estimates of Rt based on the three reported mean serial-intervals of COVID-19 all started to decline on March 19, when SAHO was first implemented in the USA, and declined faster after March 23 (Fig. 3). After a short plateau, Rt continued to decline after April 3 and fell below/around 1.0 on April 13.

We found that New York state and its neighbor states had higher daily new cases and deaths than other states (Table 2 and Fig. 4). The multivariable piecewise log-linear regression also revealed the state's neighboring relationship with New York was linked to COVID-19 daily new cases (coefficient $=-1.43,95 \%$ CI of -1.57 to -1.28 ) and new deaths (coefficient $=-1.42,95 \% \mathrm{CI}$ of -1.57 to -1.26 ), whereas the two turning points were March 28 (slope changes $=-0.09,95 \% \mathrm{CI}$ of -0.17 to -0.01 ) and April 3 (slope changes $=-0.09,95 \% \mathrm{CI}$ of -0.17 to -0.01$)$ for daily new cases, and April 9 (slope changes $=-0.06,95 \%$ CI of -0.10 to -0.02 ) and April 19 (slope changes $=-0.90,95 \%$ CI of -0.94 to -0.86 ) for daily new deaths, respectively. Correspondingly, there were 159 million USA residents ( $48.5 \%$ of the U.S. population, 23 states) under SAHO on March 28, 279.5 million U.S. residents (85.2\% of the U.S. population, 38 states) under SAHO on April 3, and 290.8 million U.S. residents $(88.6 \%$ of the U.S. population, 41 states) under SAHO since April 7.

\section{Discussion}

The population under SAHO in the USA grew from March 19,
2020 to April 6, 2020, and reached 290,829,980 (88.6\%) by April 7. The multivariable piecewise log-linear regression models identified two turning points of COVID-19 daily new cases and the time-varying reproduction number, Rt, in the USA, as well as the link between states' relationship with New York and COVID-19 epidemics. The two turning points were associated with implementation of SAHO on March 28 (affecting 48.5\% of USA population in 22 states and DC) and the CDC's face-masking recommendation on April 3. Similar turning points of COVID-19 daily new deaths were April 3 and April 15, which represented 10 and 12 days of delay, respectively. Simulation on early-implementation and lift of SAHO also reveals considerable impacts on COVID-19 daily new cases and deaths.

Several recent studies showed that public health interventions, including school closure, cordons sanitaire, traffic restriction, social distancing and others, were linked to reduction of Rt and daily incidence of COVID-19. ${ }^{8-11}$ However, these data were mostly from other countries where the socioeconomic systems and the modalities and extents of public health interventions were different from those in the USA. ${ }^{5,6}$ Among several modalities of social distancing, including school closure, gathering restrictions and restaurant restriction, ${ }^{6}$ the implementing time of SAHO appeared to be the only factor that matched to the identified turning point (March 28) in this study. Moreover, the Berkeley Interpersonal Contact Study also showed reduction in interpersonal contact in the USA between Mach 22 and April 8. ${ }^{22}$ Therefore, our and others' data together suggest that the implementation of SAHO, which is the strictest social distancing modality, may be required to reach the turning point/timing in trends of new cases. In addition, the studies in China also support our findings that travel restriction alone would reduce SARS-CoV2 transmission, ${ }^{8-10}$ but additional reduction in transmission in the community (e.g., facemasking in the USA) may be required to attenuate or reverse the epidemic trajectory.

The effects of masking on the epidemic of COVID-19 in the USA was simulated using the filtering efficacy of masks on influenza. $^{23,24}$ The influence of public intervention on COVID-19 
Table 1. Characteristics of the states that implemented a stay-at-home order (SAHO) and those not in the USA, sorted by the starting date

\begin{tabular}{|c|c|c|c|c|c|c|}
\hline State & Start date & $\begin{array}{l}\text { State popula- } \\
\text { tion", million }\end{array}$ & $\begin{array}{l}\text { Proportion of the USA population } \\
\text { under SAHO on the start date }\end{array}$ & End date* & $\begin{array}{l}\text { SAHO dura- } \\
\text { tion, days }\end{array}$ & $\begin{array}{l}\text { Total state } \\
\text { count }\end{array}$ \\
\hline California & 19-Mar & 39.5 & $12.0 \%$ & & & 1 \\
\hline Illinois & 21-Mar & 12.7 & $18.6 \%$ & & & 2 \\
\hline New Jersey & 21-Mar & 8.9 & $18.6 \%$ & & & 3 \\
\hline New York & 22-Mar & 19.5 & $24.5 \%$ & & & 4 \\
\hline Connecticut & 23-Mar & 3.6 & $34.7 \%$ & & & 5 \\
\hline Louisiana & 23-Mar & 4.6 & $34.7 \%$ & 15-May & 53 & 6 \\
\hline Ohio & 23-Mar & 11.7 & $34.7 \%$ & & & 7 \\
\hline Oregon & 23-Mar & 4.2 & $34.7 \%$ & & & 8 \\
\hline Washington & 23-Mar & 7.6 & $34.7 \%$ & & & 9 \\
\hline West Virginia & 23-Mar & 1.8 & $34.7 \%$ & 4-May & 42 & 10 \\
\hline Delaware & 24-Mar & 1.0 & $38.7 \%$ & & & 11 \\
\hline Michigan & 24-Mar & 10.0 & $38.7 \%$ & & & 12 \\
\hline New Mexico & 24-Mar & 2.1 & $38.7 \%$ & & & 13 \\
\hline Hawaii & 25-Mar & 1.4 & $43.7 \%$ & & & 14 \\
\hline Idaho & 25-Mar & 1.8 & $43.7 \%$ & 30-Apr & 36 & 15 \\
\hline Indiana & 25-Mar & 6.7 & $43.7 \%$ & 1-May & 37 & 16 \\
\hline Vermont & 25-Mar & 0.6 & $43.7 \%$ & & & 17 \\
\hline Wisconsin & 25-Mar & 5.8 & $43.7 \%$ & 13-May & 49 & 18 \\
\hline Colorado & 26-Mar & 5.8 & $45.5 \%$ & 26-Apr & 31 & 19 \\
\hline Minnesota & 27-Mar & 5.6 & $47.6 \%$ & & & 20 \\
\hline New Hampshire & 27-Mar & 1.4 & $47.6 \%$ & & & 21 \\
\hline Alaska & 28-Mar & 0.7 & $48.5 \%$ & & & 22 \\
\hline Montana & 28-Mar & 1.1 & $48.5 \%$ & 26-Apr & 29 & 23 \\
\hline Rhode Island & 28-Mar & 1.1 & $48.5 \%$ & & & 24 \\
\hline District of Columbia & 30-Mar & 0.7 & $54.6 \%$ & & & 25 \\
\hline Kansas & 30-Mar & 2.9 & $54.6 \%$ & 4-May & 35 & 26 \\
\hline Maryland & 30-Mar & 6.0 & $54.6 \%$ & 15-May & 46 & 27 \\
\hline North Carolina & 30-Mar & 1.9 & $54.6 \%$ & & & 28 \\
\hline Virginia & 30-Mar & 8.5 & $54.6 \%$ & & & 29 \\
\hline Alabama & 31-Mar & 4.9 & $58.3 \%$ & 30-Apr & 30 & 30 \\
\hline Arizona & 31-Mar & 7.3 & $58.3 \%$ & & & 31 \\
\hline Nevada & 1-Apr & 3.1 & $63.2 \%$ & & & 32 \\
\hline Pennsylvania & 1-Apr & 12.8 & $63.2 \%$ & & & 33 \\
\hline Maine & 2-Apr & 1.3 & $74.5 \%$ & 30-Apr & 28 & 34 \\
\hline Tennessee & 2-Apr & 6.8 & $74.5 \%$ & 30-Apr & 28 & 35 \\
\hline Texas & 2-Apr & 29.0 & $74.5 \%$ & 30-Apr & 28 & 36 \\
\hline Florida & 3-Apr & 21.5 & $85.2 \%$ & 4-May & 31 & 37 \\
\hline Georgia & 3-Apr & 10.6 & $85.2 \%$ & 30-Apr & 27 & 38 \\
\hline Mississippi & 3-Apr & 3.0 & $85.2 \%$ & 27-Apr & 24 & 39 \\
\hline Missouri & 6-Apr & 6.1 & $87.0 \%$ & 3-May & 27 & 40 \\
\hline South Carolina & 7-Apr & 5.1 & $88.6 \%$ & 4-May & 27 & 41 \\
\hline
\end{tabular}


$\mathrm{Xu}$ J. et al: Policy and COVID-19 trends

Explor Res Hypothesis Med

Table 1. Characteristics of the states that implemented a stay-at-home order (SAHO) and those not in the USA, sorted by the starting date - (continued)

\begin{tabular}{|c|c|c|c|c|c|c|}
\hline State & Start date & $\begin{array}{l}\text { State popula- } \\
\text { tion\#, million }\end{array}$ & $\begin{array}{l}\text { Proportion of the USA population } \\
\text { under SAHO on the start date }\end{array}$ & End date* & $\begin{array}{l}\text { SAHO dura- } \\
\text { tion, days }\end{array}$ & $\begin{array}{l}\text { Total state } \\
\text { count }\end{array}$ \\
\hline Arkansas & NA & 3.0 & & & & \\
\hline lowa & NA & 3.2 & & & & \\
\hline Kentucky & NA & 4.5 & & & & \\
\hline Massachusetts & NA & 6.9 & & & & \\
\hline North Dakota & NA & 10.5 & & & & \\
\hline Nebraska & NA & 0.8 & & & & \\
\hline Oklahoma & NA & 4.0 & & & & \\
\hline South Dakota & NA & 0.9 & & & & \\
\hline Utah & NA & 3.2 & & & & \\
\hline Wyoming & NA & 0.6 & & & & \\
\hline
\end{tabular}

\#The state populations were extracted from the Census data up to July 2019. *Only the statewide lift of SAHO was counted. Some states used a three-phase reopening strategy but have not reached completely open status as of July 1, 2020. Some counties elected to lift the SAHO before the statewide lift was announced. NA, not applicable due to no statewide SAHO.
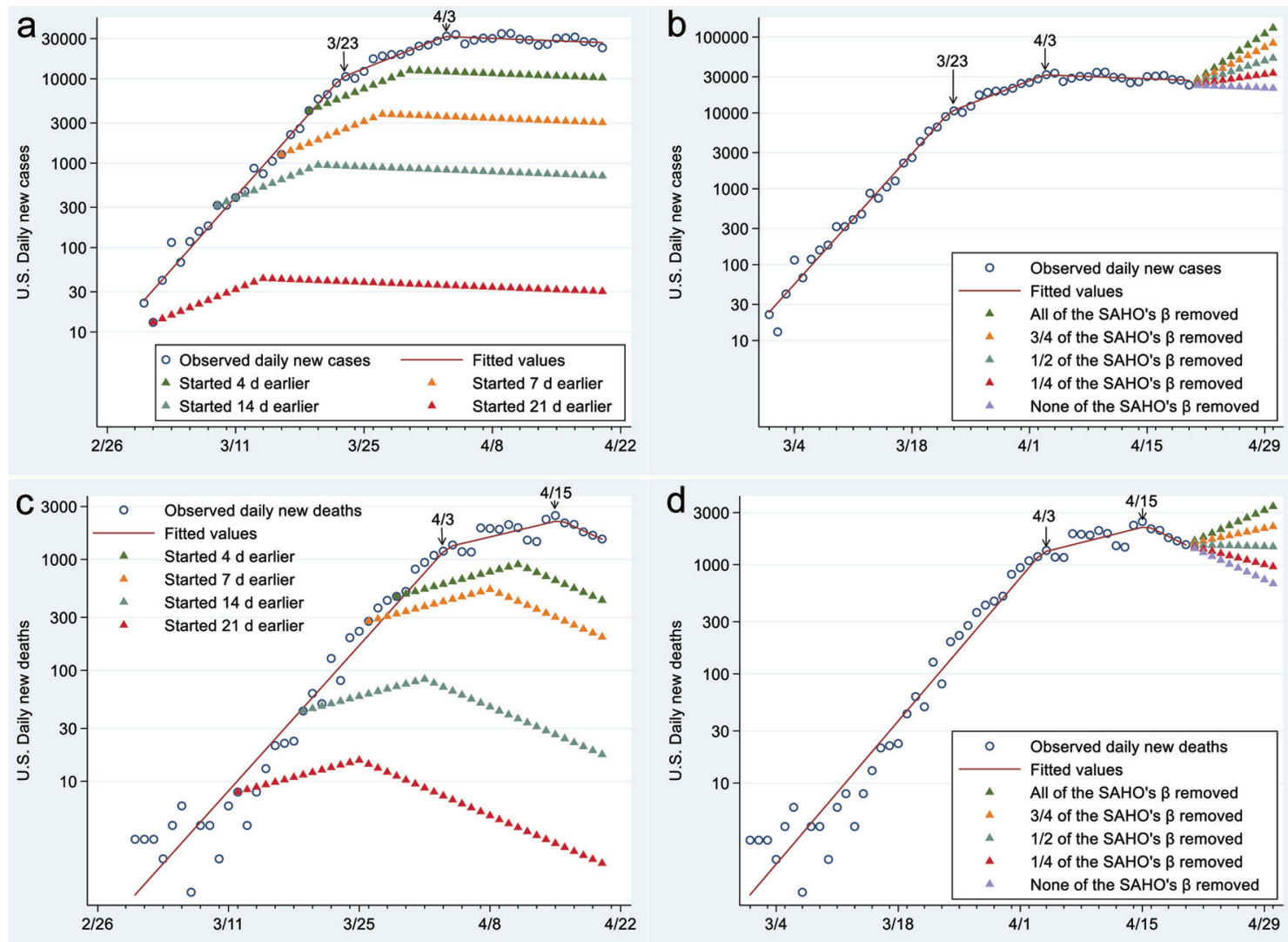

Fig. 2. Observed and simulated trends in daily new cases and deaths of laboratory-confirmed COVID-19 in the USA between March 1 and April 30, 2020. The Joinpoint analyses with Poisson variance model shows that the two turning points of March 23 and April 3 divided the trends in USA COVID-19 daily new cases into three segments, with the coefficients of $31.69(95 \% \mathrm{Cl}$ of 26.82 to $36.75, p<0.001), 9.75$ ( $95 \% \mathrm{Cl}$ of 7.54 to $12.01, p<0.001)$, and -0.90 ( $95 \%$ $\mathrm{Cl}$ of -1.62 to $-0.17, p=0.02$ ), respectively. These turning points appeared to link to implementation of a SAHO by 10 states on March 23 , and the CDC's face-masking recommendation on April 3. Similarly, the two turning points of April 3 and April 15 divided the trends in USA COVID-19 daily new deaths into three segments, with the coefficients of 25.06 (95\% Cl of 21.44 to $28.79, p<0.001), 5.22(95 \% \mathrm{Cl}$ of 3.36 to $7.11, p<0.001)$, and -7.90 (95\% $\mathrm{Cl}$ of -13.45 to $-1.99, p=0.01)$, respectively. The simulated results on early-implementations of SAHO and face-masking recommendation and early-lift of SAHO are shown in A and C, and B and D, respectively, in a form removing a portion or all of the slope changes attributable to SAHO (denoted as removal of SAHO'S coefficient(ß) in the figure). 

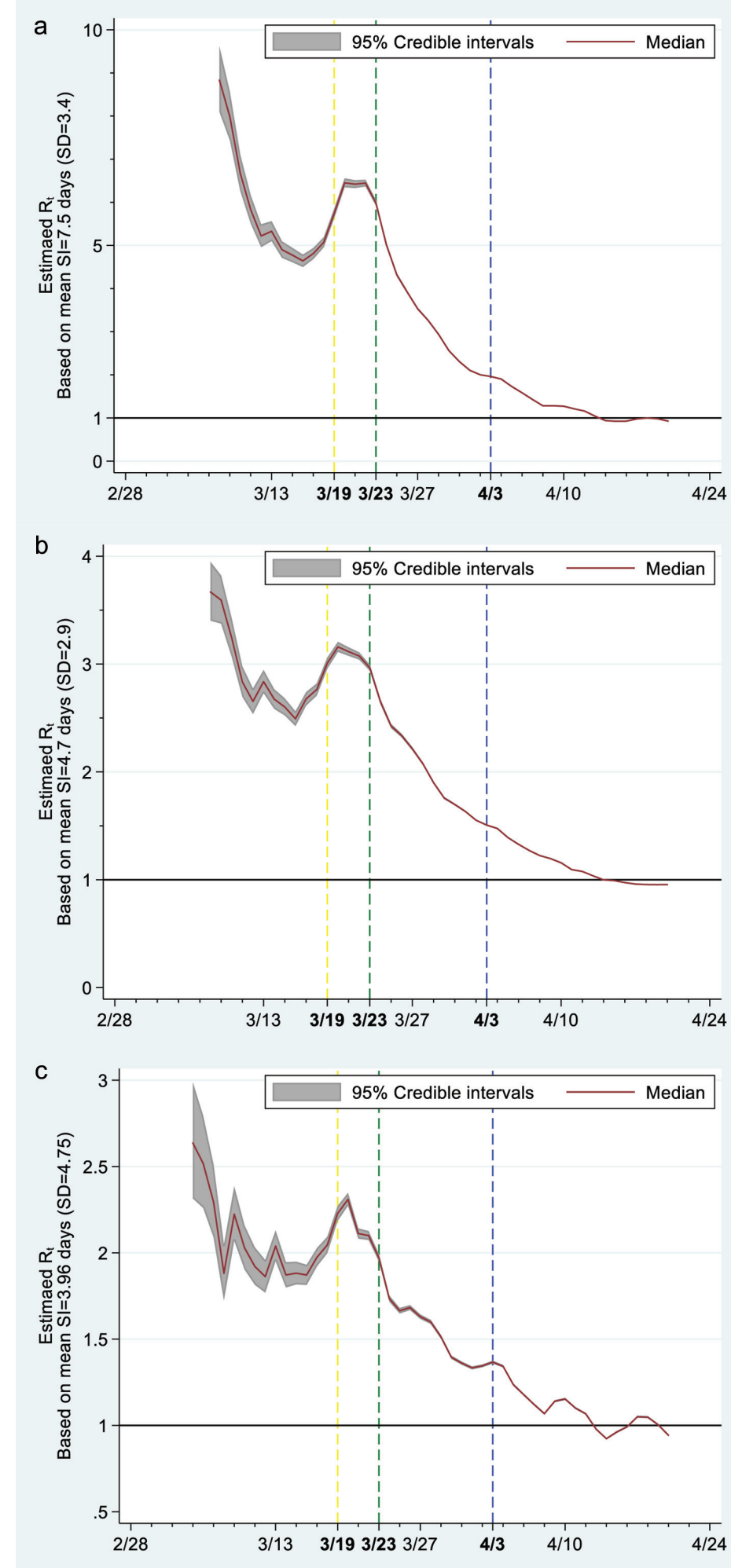

Fig. 3. Estimated effective Rt based on laboratory-confirmed COVID-19 cases in the USA and the reported serial intervals. The effective Rt was estimated using the previously-reported COVID-19 mean serial-intervals (SI) of 7.5 (A), 4.7 (B) and 3.96 (C) days, as well as the corresponding standard deviations (SDs). The statewide SAHO was first implemented by the state of California on March 19, 2020 (yellow dashed line). Ten states had implemented a SAHO by March 23, 2020 (green dashed line), affecting 114,047,753 residents (37.45\% of the U.S. population). The CDC recommended face-masking on April 3, 2020 (blue dashed line). These dates were linked to the declines in Rt's at the times of an increase or plateau of the Rt. 
Table 2. COVID-19 epidemics by the states' neighboring relationship with New York in the USA, March 1 to April 20, 2020.

\begin{tabular}{|c|c|c|c|c|c|c|}
\hline State category\# & $\begin{array}{l}\text { Daily new cases, } \\
\text { median (interquartile) }\end{array}$ & Total cases & $p$-value* & $\begin{array}{l}\text { Daily new deaths, } \\
\text { median (interquartile) }\end{array}$ & $\begin{array}{l}\text { Total } \\
\text { deaths }\end{array}$ & $p$-value* \\
\hline New York & $6,392(682$ to 8,327$)$ & 247,506 & & 209 (0 to 606) & 14,347 & \\
\hline Neighbor states of New York, $n=6$ & 179 (20 to 1,178$)$ & 185,830 & $<0.001$ & 2 (0 to 27$)$ & 8,811 & $<0.001$ \\
\hline Other states, $n=34$ & 64 (14 to 209$)$ & 337,629 & $<0.001$ & 1 (0 to 6$)$ & 14,086 & $<0.001$ \\
\hline
\end{tabular}

\#The District of Columbia was included. *The $p$-values of comparing the state groups with New York (Wilcoxon rank sum and Student's $t$ test) were all smaller than 0.001.

mortalities in New York and the USA were also simulated using mathematical models and the data of COVID-19, Ebola and influenza viruses. ${ }^{25}$ However, neither of the studies used laboratoryconfirmed cases and COVID-19-based models. Further, neither of the studies simulated the early implementation of SAHO. Therefore, using the population data of laboratory-confirmed cases, we simulated the potential outcomes of implementing SAHO and face-masking recommendation based on the piecewise log-linear
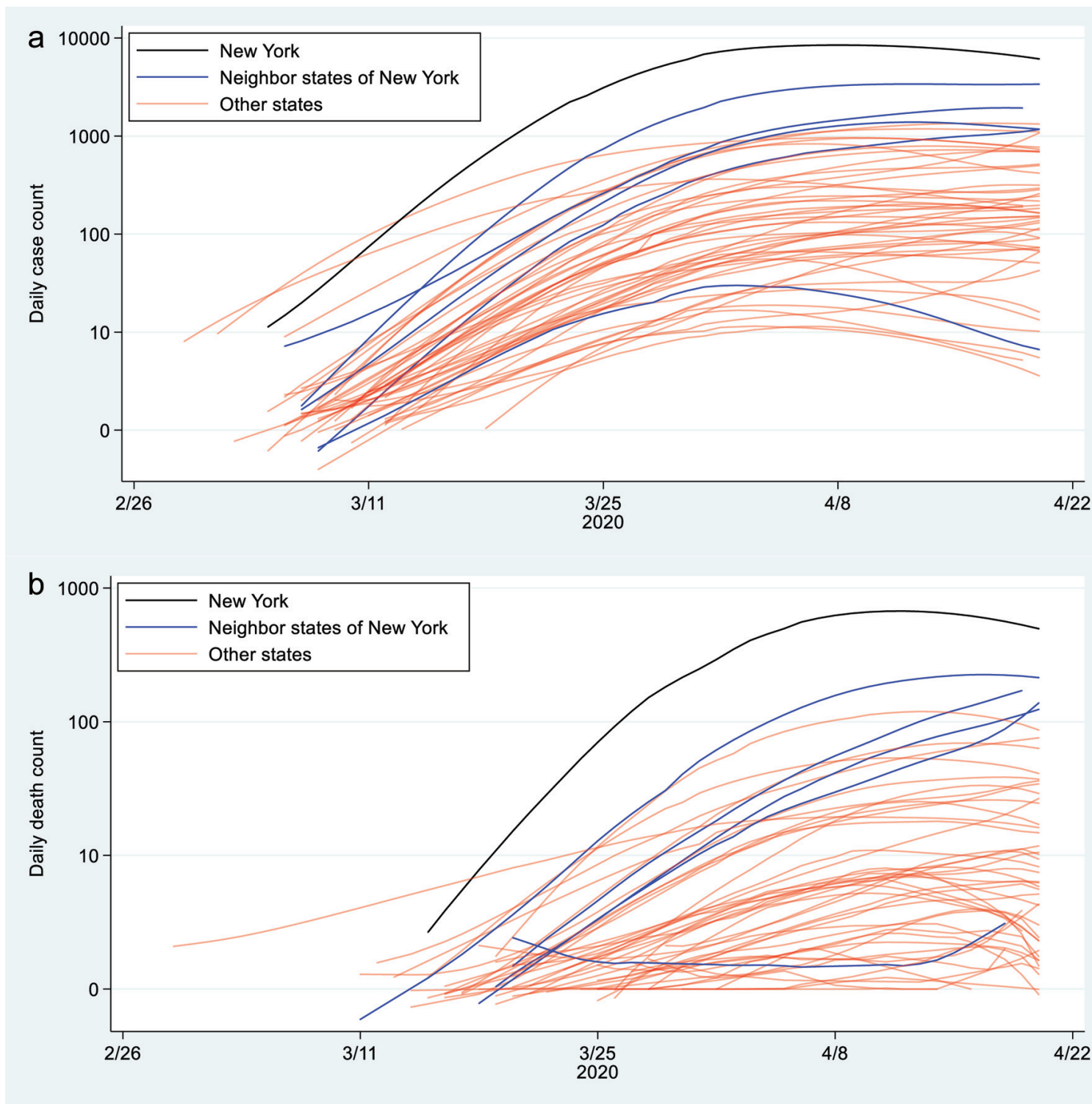

Fig. 4. Observed trends in daily new cases and deaths of laboratory-confirmed COVID-19 by state neighboring relationship with New York in the USA between March 1 and April 30, 2020. The daily new cases (A) and new deaths (B) of COVID-19 were higher in New York than in the six neighboring states of New York (Connecticut, Massachusetts, New Jersey, Pennsylvania, Rhode Island, and Vermont), and higher in the neighboring states than other non-New York states ( $p<0.001$ for both). The lines were smoothed using a locally weighted scatter smoothing (known as LOWESS) algorithm. 
models recommended by the guidelines of the USA's National Center for Health Statistics and other methodological considerations. ${ }^{26-29}$ The simulation analysis demonstrates a much smaller scale of COVID-19 in the USA if SAHO would have been implemented earlier, and a concerning reverse of stable downward trends in COVID-19 daily new cases and deaths if being lifted soon. Indeed, the SAHO was not implemented in any of the USA states till March 19, when the daily new cases reached 4,190 (crude daily incidence of 12.8 per 1 million) in the USA. ${ }^{1}$ In contrast, a much more strict SAHO was implemented in China on January 23, when the daily new cases were 259 (crude daily incidence of 0.18 per 1 million) in China and 70 (crude daily incidence of 7.2 per 1 million) in Wuhan. ${ }^{8,30}$ These data suggest an earlier implementation of SAHO in terms of crude daily incidence would be more effective. Consistent with the previous study and recent surge in daily new cases, ${ }^{25,31}$ we also show that (early) lift of SAHO would be associated with a second wave or upward trend of COVID-19 daily new cases and deaths. Therefore, caution should be used when considering lift of SAHO prematurely.

$\mathrm{Rt}$ is one of the most widely used metrics for assessing transmission rate of infectious diseases, ${ }^{8,25}$ and linked to the incidence decay with exponential adjustment model (known as IDEA) and Farr's law. ${ }^{32}$ However, Rt is difficult to estimate. One of the challenges is the variances in the SARS-CoV2 serial intervals. ${ }^{15-17}$ The reported serial intervals of COVID-19 ranged from 3.96 to 7.5 days, and were first used to rigorously examine the changes of Rt associated with SAHO and face-masking recommendation in the USA. Two similar turning points were identified in the Rt trends estimated using three different serial intervals, and further support the findings discovered using our piecewise log-linear model.

Geographic attributes have been shown to be linked to COVID-19 epidemics in several countries. ${ }^{33-36}$ The geographic differences of COVID-19 incidence and deaths in states have been reported in a previous study, ${ }^{37}$ which, unfortunately, did not conduct quantitative trend analyses. The states' neighboring relationship with the epicenter (New York) in the USA was linked to the COVID-19 epidemics in our multivariable models, while the number of states under SAHO, proportion of positive COVID-19 testing and the number and proportion of the populations under SAHO were not linked to them. Additional works are needed to better understand the geographic differences in state-level COVID-19 trends.

Many COVID-19 studies were published as preprints. Some of them were of inferior methodological merit and low reporting quality, as discussed before. ${ }^{30,38,39}$ This observation is concerning, and calls for more collaborative efforts in the efficient review and timely dissemination of studies on COVID-19.

The major strength of this study is that we used state-level and national data of laboratory-confirmed cases in the analyses. ${ }^{2}$ Moreover, a very rigorous study-design was used. We applied multivariable models to exclude confounders and a quasi-experimental interrupted time series study-design to infer the impacts of public health interventions. The quasi-experimental interrupted time series study-design is likely the most rigorous method available for when randomization and controlling are not possible, ${ }^{12,13,19}$ as the case for when COVID-19 SAHO and face-masking were implemented in the USA. Further, two piecewise log-linear regression methods were used to rigorously examine trends changes, according to the guidelines on trend analyses of population data and other methodological considerations. ${ }^{26-29}$ Finally, the simulation analysis provides comprehensive estimates of trends changes linked to early-implementation of SAHO at various time points and early-lift of SAHO with various extents. Indeed, the recent upward trends in the USA COVID-19 epidemics are consistent with our simulated scenarios of early SAHO lift (even in some states or counties). ${ }^{2,3,31}$ Thus, the proper timing and duration of SAHO implementation warrant further research.

Several limitations of this study are noteworthy. First, the COVID-19 positive rates varied among states and by time, suggesting under-testing of the potential patients. The exact COVID-19 case numbers are thus not available, although efforts were made to estimate them using the Johns Hopkins' data repository of COVID-19 cases $^{40}$ Given the data inconsistence we noticed, ${ }^{1}$ such an estimation was not optimal in our view. We were more confident in the reliability of the laboratory-confirmed case numbers. Inclusion of positive-test rate in our models may alleviate the variances in test rate across time. Indeed, the positive-test rate was not a factor linked to COVID-19 epidemics in our multivariable models. Second, there was a lag in COVID-19 reporting, ${ }^{41}$ which may lead to inaccurate estimation of the case numbers. However, the increases in the proportion of the tested population appeared stable in the USA, ${ }^{2}$ and was not a factor linked to COVID-19 epidemics in our multivariable models. It suggests that the lag in reporting may not change significantly as the time changes, and will have minimal impact on the trend analyses. Third, the CDC changed the diagnostic criteria of COVID-19 on April 14, 2020. The timing and extent of these changes in each state were largely unknown, while we consider the data released before April 20 acceptable owing to the transition time of different criteria. Therefore, the data released after April 20 would have an unmeasurable bias and would not be suitable for comparing with those before April 20. Thus, we were not able to reliably compare the observed and the simulated COVID-19 trends after April 20. Finally, we did not report the daily incidence. There were no significant changes in the USA population during the study-period. The daily new cases of COVID-19 thus should be proportional to its daily incidence in the USA but are easier to interpret than daily incidence and were used here.

\section{Future direction}

Future research should be focused on how state-level SAHO changes the COVID-19 epidemics. This is particularly interesting since state heterogeneity has been reported in 27 European Union states, ${ }^{19}$ and in the USA, states' neighboring relationship with New York was linked to COVID-19 epidemics (as this study showed). As more data and more-reliable data become available, we will keep looking into the association of SAHO and face-masking policies with changes in the COVID-19 epidemic.

\section{Conclusions}

There were two turning points of COVID-19 daily new cases or deaths in the USA, which appeared to be associated with the implementation of SAHO and the CDC's face-masking recommendation. Simulation on early-implementation and lift of SAHO reveals considerable impact of these interventions on COVID-19 daily new cases and deaths. These findings may inform decision-making of lifting SAHO and face-masking recommendation.

\section{Acknowledgments}

This work has been deposited at MedRxiv https://doi.org/10.1101 /2020.05.01.20088237. 
Data sharing statement

All raw data used here are publicly available at their respective websites. No additional data are available.

\section{Funding}

The work was supported in part by a grant from the National Institutes of Health (R03 HD100708 to WB).

\section{Conflict of interest}

No disclosures were reported.

\section{Author contributions}

Supervision (LZ), concept and design (JX, LZ), drafting of the manuscript (JX), statistical analysis (LZ), acquisition, analysis, or interpretation of data (JX, SH, GL, KZ, SW, WB, LZ), critical revision of the manuscript for important intellectual content (all authors). Dr. Zhang (LZ) had full access to all of the data in the study and takes responsibility for the integrity of the data and the accuracy of the data analysis.

\section{References}

[1] Yuan X, Xu J, Hussain S, Wang H, Gao N, Zhang L. Trends and Prediction in Daily New Cases and Deaths of COVID-19 in the United States: An Internet Search-Interest Based Model. Explor Res Hypothesis Med 2020;5(2):1-6. doi:10.14218/ERHM.2020.00023.

[2] COVID-Tracking. The COVID Tracking Project. Available from: https:// covidtracking.com/about-data. Accessed July 3, 2020.

[3] CDC. Cases of Coronavirus Disease (COVID-19) in the U.S. Available from: https://www.cdc.gov/coronavirus/2019-ncov/cases-updates/ cases-in-us.html. Accessed July 3, 2020.

[4] Zhang L. Blind Spots in Fighting the Outbreak of Coronavirus Disease 2019. Explor Res Hypothesis Med 2020;5(1):6-7. doi:10.14218/ ERHM.2020.00012.

[5] Wikipedia. U.S. state and local government response to the COVID-19 pandemic. Available from: https://en.wikipedia.org/wiki/U.S._state_ and local government response to the COVID-19 pandemic. Accessed July 5, 2020.

[6] Adolph C, Amano K, Bang-Jensen B, Fullman N, Wilkerson J. Pandemic Politics: Timing State-Level Social Distancing Responses to COVID-19. APSA Preprints 2020. doi:10.33774/apsa-2020-sf0ps.

[7] CDC.Recommendation RegardingtheUseofClothFaceCoverings, Especially in Areas of Significant Community-Based Transmission. Available from: https://www.cdc.gov/coronavirus/2019-ncov/prevent-gettingsick/cloth-face-cover.html. Accessed April 16, 2020.

[8] Pan A, Liu L, Wang C, Guo H, Hao X, Wang Q, et al. Association of Public Health Interventions With the Epidemiology of the COVID-19 Outbreak in Wuhan, China. JAMA 2020;323(19):1-9. doi:10.1001/ jama.2020.6130.

[9] Zhang J, Litvinova $\mathrm{M}$, Liang $\mathrm{Y}$, Wang $\mathrm{Y}$, Wang W, Zhao S, et al. Changes in contact patterns shape the dynamics of the COVID-19 outbreak in China. Science 2020;368(6498):1481-1486. doi:10.1126/science. abb8001.

[10] Chinazzi M, Davis JT, Ajelli M, Gioannini C, Litvinova M, Merler S, et al. The effect of travel restrictions on the spread of the 2019 novel coronavirus (COVID-19) outbreak. Science 2020;368(6489):395-400. doi:10.1126/science.aba9757

[11] Jüni $P$, Rothenbühler $M$, Bobos $P$, Thorpe $K E$, da Costa BR, Fis- man DN, et al. Impact of climate and public health interventions on the COVID-19 pandemic: a prospective cohort study. CMAJ 2020;192(21):E566-E573. doi:10.1503/cmaj.200920.

[12] Reeves BC, Wells GA, Waddington H. Quasi-experimental study designs series-paper 5: a checklist for classifying studies evaluating the effects on health interventions-a taxonomy without labels. J Clin Epidemiol 2017:89:30-42. doi:10.1016/j.jclinepi.2017.02.016.

[13] Kontopantelis E, Doran T, Springate DA, Buchan I, Reeves D. Regression based quasi-experimental approach when randomisation is not an option: interrupted time series analysis. BMJ 2015;350:h2750. doi:10.1136/bmj.h2750.

[14] Census. State Population Totals and Components of Change: 20102019. Available from: https://www.census.gov/data/tables/time-series/demo/popest/2010s-state-total.html. Accessed April 20, 2030.

[15] Li Q, Guan X, Wu P, Wang X, Zhou L, Tong Y, et al. Early Transmission Dynamics in Wuhan, China, of Novel Coronavirus-Infected Pneumonia. N Engl J Med 2020;382(13):1199-1207. doi:10.1056/ NEJMoa2001316.

[16] Nishiura H, Linton NM, Akhmetzhanov AR. Serial interval of nove coronavirus (COVID-19) infections. Int J Infect Dis 2020;93:284-286. doi:10.1016/j.ijid.2020.02.060.

[17] Du Z, Xu X, Wu Y, Wang L, Cowling BJ, Meyers LA. Serial Interval of COVID-19 among Publicly Reported Confirmed Cases. Emerg Infect Dis 2020;26(6):1341-1343. doi:10.3201/eid2606.200357.

[18] Cori A, Ferguson NM, Fraser C, Cauchemez S. A new framework and software to estimate time-varying reproduction numbers during epidemics. Am J Epidemiol 2013;178(9):1505-1512. doi:10.1093/aje/ kwt133.

[19] Yuan X, Hu K, Xu J, Zhang X, Bao W, Lynch CF, et al. State heterogeneity of human mobility and COVID-19 epidemics in the European Union. medRxiv 2020:20127530. doi:10.1101/2020.06.10.20127530.

[20] Cox NJ. Speaking Stata: Smoothing in various directions. Stata Journal 2005:5(4):574-593. doi:10.1177/1536867X0500500408.

[21] Cleveland WS. Robust locally weighted regression and smoothing scatterplots. J Am Stat Assoc 1979;74(368):829-836. doi:10.1080/0 1621459.1979.10481038.

[22] Feehan D, Mahmud A. Quantifying interpersonal contact in the United States during the spread of COVID-19: first results from the Berkeley Interpersonal Contact Study. medRxiv 2020:20064014. doi: 10.1101/2020.04.13.20064014.

[23] Eikenberry SE, Mancuso M, Iboi E, Phan T, Eikenberry K, Kuang Y, et al. To mask or not to mask: Modeling the potential for face mask use by the general public to curtail the COVID-19 pandemic. medRxiv 2020:20055624. doi:10.1101/2020.04.06.20055624.

[24] Davies A, Thompson KA, Giri K, Kafatos G, Walker J, Bennett A. Testing the efficacy of homemade masks: would they protect in an influenza pandemic? Disaster Med Public Health Prep 2013;7(4):413418. doi:10.1017/dmp.2013.43.

[25] Ngonghala CN, Iboi E, Eikenberry S, Scotch M, MacIntyre CR, Bonds $\mathrm{MH}$, et al. Mathematical assessment of the impact of non-pharmaceutical interventions on curtailing the 2019 novel Coronavirus. Math Biosci 2020;325:108364. doi:10.1016/j.mbs.2020.108364.

[26] Ingram DD, Malec DJ, Makuc DM, Kruszon-Moran D, Gindi RM, Albert $M$, et al. National Center for Health Statistics Guidelines for Analysis of Trends. National Center for Health Statistics. Vital and Health Statistics (series 2) 2018(179):1-71. Available from: https://www.cdc. gov/nchs/data/series/sr 02/sr02 179.pdf. Accessed July 5, 2020.

[27] Xu J, Lin Y, Yang M, Zhang L. Statistics and pitfalls of trend analysis in cancer research: a review focused on statistical packages. J Cancer 2020;11(10):2957-2961. doi:10.7150/jca.43521.

[28] Zhang J, Lin Y, Zhang L. Trends in Alcoholic Fatty Liver Disease. JAMA 2019;322(10):979-980. doi:10.1001/jama.2019.10347.

[29] Yuan X, Song F, Zhang L. Trend analysis of diabetic mortality. Lancet 2019;393(10184):1931-1932. doi:10.1016/S0140-6736(18)33051-4.

[30] Xu J, Cheng Y, Yuan X, Li WV, Zhang L. Trends and prediction in daily incidence of novel coronavirus infection in China, Hubei Province and Wuhan City: an application of Farr's law. Am J Transl Res 2020;12(4):1355-1361.

[31] CDC. COVIDView: A Weekly Surveillance Summary of U.S. COVID-19 Activity. Available from: https://www.cdc.gov/coronavirus/2019ncov/covid-data/covidview/index.html. Accessed July 3, 2020 
[32] Santillana M, Tuite A, Nasserie T, Fine P, Champredon D, Chindelevitch $\mathrm{L}$, et al. Relatedness of the incidence decay with exponential adjustment (IDEA) model, "Farr's law" and SIR compartmental difference equation models. Infect Dis Model 2018;3:1-12. doi:10.1016/j. idm.2018.03.001.

[33] Wang KW, Gao J, Wang H, Wu XL, Yuan QF, Guo FY, et al. Epidemiology of 2019 novel coronavirus in Jiangsu Province, China after wartime control measures: A population-level retrospective study. Travel Med Infect Dis 2020;35:101654. doi:10.1016/j.tmaid.2020.101654.

[34] Khose S, Moore JX, Wang HE. Epidemiology of the 2020 Pandemic of COVID-19 in the State of Texas: The First Month of Community Spread. J Community Health 2020;45(4):696-701. doi:10.1007/s10900020-00854-4.

[35] Jen TH, Chien TW, Yeh YT, Lin JJ, Kuo SC, Chou W. Geographic risk assessment of COVID-19 transmission using recent data: An observational study. Medicine (Baltimore) 2020;99(24):e20774. doi:10.1097/ MD.0000000000020774.

[36] Gupta S, Raghuwanshi GS, Chanda A. Effect of weather on COVID-19 spread in the US: A prediction model for India in 2020. Sci Total Environ 2020;728:138860. doi:10.1016/j.scitotenv.2020.138860.

[37] CDC COVID-19 Response Team. Geographic Differences in COVID-19 Cases, Deaths, and Incidence - United States, February 12-April 7, 2020. MMWR Morb Mortal Wkly Rep 2020;69(15):465-471. doi:10.15585/mmwr.mm6915e4.

[38] Maslove DM. Medical Preprints-A Debate Worth Having. JAMA 2018;319(5):443-444. doi:10.1001/jama.2017.17566.

[39] Krumholz HM, Ross JS, Otto CM. Will research preprints improve healthcare for patients? BMJ 2018;362:k3628. doi:10.1136/bmj. k3628.

[40] Thorpe DG, Lyberger K. Estimating the number of SARS-CoV-2 infections in the United States. medRxiv 2020:20064519. doi:10.1101/20 20.04.13.20064519.

[41] Buchanan L, Lai KKR, Allison McCann A. U.S. lags in coronavirus testing after slow response to outbreak. Available from: https://www. nytimes.com/interactive/2020/03/17/us/coronavirus-testing-data. html. Accessed April 16, 2020. 